\title{
Dynamic Analysis of a Two-Language Competitive Model with Control Strategies
}

\author{
Lin-Fei Nie, ${ }^{1,2}$ Zhi-Dong Teng, ${ }^{1}$ Juan J. Nieto, ${ }^{3,4}$ and Il Hyo Jung ${ }^{2}$ \\ ${ }^{1}$ College of Mathematics and Systems Science, Xinjiang University, Urumqi 830046, China \\ ${ }^{2}$ Department of Mathematics, Pusan National University, Busan 609-735, Republic of Korea \\ ${ }^{3}$ Departamento de Análisis Matemático and Instituto de Matemáticas, Universidade de Santiago de Compostela, \\ 15782 Santiago de Compostela, Spain \\ ${ }^{4}$ Department of Mathematics, Faculty of Science, King Abdulaziz University, Jeddah 21589, Saudi Arabia
}

Correspondence should be addressed to Lin-Fei Nie; lfnie@163.com

Received 2 August 2013; Accepted 7 October 2013

Academic Editor: Bashir Ahmad

Copyright (C) 2013 Lin-Fei Nie et al. This is an open access article distributed under the Creative Commons Attribution License, which permits unrestricted use, distribution, and reproduction in any medium, provided the original work is properly cited.

The dynamic behavior of a two-language competitive model is analyzed systemically in this paper. By the linearization and the Bendixson-Dulac theorem on dynamical system, some sufficient conditions on the globally asymptotical stability of the trivial equilibria and the existence and the stability of the positive equilibrium of this model are presented. Nextly, in order to protect the endangered language, an optimal control problem relative to this model is explored. We derive some necessary conditions to solve the optimal control problem and present some numerical simulations using a Runge-Kutta fourth-order method. Finally, the languages competitive model is extended to this model assessing the impact of state-dependent pulse control strategy. Using the Poincaré map, differential inequality, and method of qualitative analysis, we prove the existence and stability of positive order-1 periodic solution for this control model. Numerical simulations are carried out to illustrate the main results and the feasibility of state-dependent impulsive control strategy.

\section{Introduction}

The diversity of cultures is the greatest charm of the human civilization, and languages are the most important carrier for culture. In the past decades, with the progress of the globalization, local tongues are increasingly replaced by hegemonic languages [1], this trend that has been investigated from multiple points of view, including that of physics. We refer to some of them in [2-11] and the references therein.

Perhaps the earliest and simplest mathematical model for languages shift was developed by Abrams and Strogatz [2], Patriarca et al. [12,13], and Stauffer et al. [14]. They considered a stable population in which two languages with different statuses competed for speakers and predicted that one of the languages would inevitably die out. The theoretical results were successfully fitted to historical data on the competition between Scottish Gaelic and English, Welsh and English, and Quechua and Spanish, among other language pairings [2]. However, there was no mention of the fact that the possibility of bilingual individuals might exist, a possibility that is of course realized in numerous multilingual societies. This is widely exists in all parts of the world. For example, in Spain, Castilian Spanish is the official language throughout the state, but in certain regions it is coofficial with another language (mainly Galician, Basque, Catalan, or Valencian); individual bilingualism is common in communities with more than one official language.

Recently, Mira et al. $[8,15,16]$ proposed a modified Abrams-Strogatz model that allows for bilingual as well as monolingual speakers of the competing languages and that includes a parameter that represents the ease of bilingualism. The model is accordingly described by the following differential equations:

$$
\begin{aligned}
& \frac{\mathrm{d} x}{\mathrm{~d} t}=y P_{Y X}+b P_{B X}-x\left(P_{X Y}+P_{X B}\right), \\
& \frac{\mathrm{d} y}{\mathrm{~d} t}=x P_{X Y}+b P_{B Y}-y\left(P_{Y X}+P_{Y B}\right), \\
& \frac{\mathrm{d} b}{\mathrm{~d} t}=x P_{X B}+y P_{Y B}-b\left(P_{B Y}+P_{B X}\right),
\end{aligned}
$$


where capital letters $X$ and $Y$ denote the two languages spoken in population; the uppercased letter $B$ denotes the group of bilingual speakers; and the lowercased letters $x, y$, and $b$ (with $x+y+b=1$ ) refer to the fraction of speakers of each of the languages in population and the fraction of bilingual speakers, respectively; parameter $P_{X Y}$ denotes the probability of a monolingual speaker of language $X$ being replaced in the population by a monolingual speaker of language $Y$, with analogous notation for the other parameters $P_{Y X}, P_{X B}, P_{B X}, P_{Y B}$, and $P_{B Y}$. The probability of a monolingual person being replaced by mono- or bilingual speaker of the other language is assumed to be proportional both to the status of the second language, that is, the social and/or economic advantages it offers, and to a power of the proportion of population that speaks it. Thus, denoting by $s$ the relative status of language $X$ and by $1-s$ that of language $Y$,

$$
\begin{gathered}
P_{X B}=c k(1-s)(1-x)^{\alpha}, \\
P_{B X}=P_{Y X}=c(1-k) s(1-y)^{\alpha}, \\
P_{Y B}=c k s(1-y)^{\alpha}, \\
P_{B Y}=P_{X Y}=c(1-k)(1-s)(1-x)^{\alpha},
\end{gathered}
$$

where $c$ is a normalization factor related to the time scale, $\alpha$ is the power parameter, and $k$ is the probability that the disappearance of a monolingual speaker of language $X$ (resp., $Y$ ) will be compensated for by the appearance of a bilingual rather than by a monolingual speaker of language $Y$ (resp., $X$ ). On basis of detailed analysis and extensive calculations, authors showed that both languages may coexist and survive in the long term. They pointed out that it is possible only if the competing languages are sufficiently similar, in which case its occurrence is favored by both similarity and status symmetry.

It is generally known that the disappearance of race language will bring the disappearance of race culture, even the whole disappearance of the corresponding race. The protection of endangered language has been concerned increasingly interdisciplinary in different contexts.

Very recently, the dynamical model with optimal control strategies has become a major topic in mathematical biology (see [17-23] and the references therein). Particularly, Joshi, in [18], proposed an HIV immunology model with optimal drug treatment strategies, and the existence and uniqueness results for the optimal control pair are established. Jung et al. [24] proposed a two-strain tuberculosis model with two control terms, and the optimal controls are characterized in terms of the optimality system. In addition, the state-dependent impulsive feedback control measure is also applied widely to the control of spread of infectious disease due to its economic, high-efficiency, and feasibility nature; see $[25,26]$ and the references therein.

Motivated by these facts, in this paper, the dynamic behavior of two-language competitive model (1) with (2) is analyzed systemically in Section 2. A set of necessary conditions that an optimal control and state must satisfy, are derived in Section 3. In Section 4, we extend model (1) with state-dependent pulse control measure. Some sufficient conditions are presented in this section for the existence and stability of positive periodic solution. Some concluding remarks are presented in Section 5.

\section{Qualitative Analysis for Model (1)}

Inserting the parameters formula (2) into model (1) and taking into account $x+y+b=1$, we get the following reduced model (here, we assume that $\alpha=1$ throughout the rest of this paper):

$$
\begin{aligned}
& \frac{\mathrm{d} x}{\mathrm{~d} t} \\
& =c s(1-k)(1-x)(1-y)-c(1-s) x(1-x):=f_{1}(x, y), \\
& \frac{\mathrm{d} y}{\mathrm{~d} t} \\
& =c(1-k)(1-s)(1-x)(1-y)-c s y(1-y):=f_{2}(x, y) .
\end{aligned}
$$

By the biological background of model (3), we only consider model (3) in the biological meaning region $\Omega=$ $\{(x, y): x \geq 0, y \geq 0, x+y \leq 1\}$.

The following theorem is on the nonnegativity of solution of model (3).

Theorem 1. The region $\Omega$ is positively invariant for model (3). here.

The proof of Theorem 1 is simple; we, therefore, omit it

Now, we discussed the existence and stability of equilibria for model (3). The isocline corresponding to $\mathrm{d} x / \mathrm{d} t=0$ is the line $s(1-k)(1-y)=(1-s) x$. The isocline corresponding to $\mathrm{d} y / \mathrm{d} t=0$ is the line $(1-k)(1-s)(1-x)=s y$. Note that two isoclines do not have positive intersection point for $s<(1-s)(1-k)$ or $(1-s)<s(1-k)$ and have only one positive intersection point for $1-k<(1-$ $s) / s<1 /(1-k)$. Namely, model (3) has only two trivial equilibria $(1,0)$ and $(0,1)$ for $s<(1-s)(1-k)$ or $(1-$ $s)<s(1-k)$ and has two trivial equilibria $(1,0) ;(0,1)$ and a positive equilibrium $E\left(x^{*}, y^{*}\right)$ for $1-k<(1-s) / s<$ $1 /(1-k)$, where

$$
\begin{aligned}
& x^{*}=\frac{(1-k) s-(1-s)(1-k)^{2}}{1-s-(1-s)(1-k)^{2}}, \\
& y^{*}=\frac{(1-k)(1-s)-(1-k)^{2} s}{s\left(1-(1-k)^{2}\right)} .
\end{aligned}
$$

The locally asymptotical stabilities of equilibria are determined by the eigenvalues of Jacobian matrixes of the linearization of model (3) around equilibria. It is easy to calculate that

$$
\begin{aligned}
& \frac{\partial f_{1}(x, y)}{\partial x} \\
& \quad=-c s(1-k)(1-y)-c(1-s)(1-x)+c(1-s) x,
\end{aligned}
$$




$$
\begin{gathered}
\frac{\partial f_{1}(x, y)}{\partial y}=-c s(1-k)(1-x), \\
\frac{\partial f_{2}(x, y)}{\partial x}=-c(1-k)(1-s)(1-y), \\
\frac{\partial f_{2}(x, y)}{\partial y}=-c(1-k)(1-s)(1-x)-c s(1-y)+c s y .
\end{gathered}
$$

Computing Jacobian matrixes from model (3) around $(1,0),(0,1)$, and $\left(x^{*}, y^{*}\right)$, we have that

$$
\begin{gathered}
J_{(1,0)}=\left(\begin{array}{cc}
c(1-s)-c s(1-k) & 0 \\
-c(1-k)(1-s) & -c s
\end{array}\right) \\
J_{(0,1)}=\left(\begin{array}{cc}
-c(1-s) & -c s(1-k) \\
0 & c s-c(1-k)(1-s)
\end{array}\right) \\
J_{\left(x^{*}, y^{*}\right)}=\left(\begin{array}{cc}
-c(1-s)\left(1-x^{*}\right) & -c s(1-k)\left(1-x^{*}\right) \\
-c(1-k)(1-s)\left(1-y^{*}\right) & -c s\left(1-y^{*}\right)
\end{array}\right) .
\end{gathered}
$$

From (6), since $(1-s)<s(1-k)$ implies that $(1-$ $s)(1-k)<s$, it follows that the two eigenvalues of $J_{(1,0)}$ are negative and that one eigenvalue is positive and another one is negative for $J_{(0,1)}$ when $(1-s)<s(1-k)$. Therefore, model (3) has a locally asymptotically stable node $(1,0)$ and a saddle point $(0,1)$ for $(1-s)<s(1-k)$. Furthermore, since there is not equilibrium in interior of $\Omega$ for $(1-s)<$ $s(1-k)$, model (3) has no periodic orbit lying entirely in the interior of $\Omega$. Therefore, $(1,0)$ is globally asymptotically stable in the interior of $\Omega$ for this case. Similarly, since $s<$ $(1-s)(1-k)$ implies that $s(1-k)<(1-s)$, we also have that $(0,1)$ is globally asymptotically stable in the interior of $\Omega$ and $(1,0)$ is a saddle point for model (3) when $s<$ $(1-s)(1-k)$.

Consider the eigenvalues of $J_{\left(x^{*}, y^{*}\right)}$; let $T=$ $\operatorname{Tr} J_{\left(x^{*}, y^{*}\right)}$ and $D=\operatorname{Det} J_{\left(x^{*}, y^{*}\right)}$ be the trace and determinant of $J_{\left(x^{*}, y^{*}\right)}$, respectively. The eigenvalues of $J_{\left(x^{*}, y^{*}\right)}$ are provided by the following characteristic equation:

$$
\lambda^{2}-T \lambda+D=0 .
$$

Note that when $(1-k)<(1-s) / s<1 /(1-k)$,

$$
\begin{aligned}
T & =-c(1-s)\left(1-x^{*}\right)-c s\left(1-y^{*}\right)<0, \\
D= & c^{2} s(1-s)\left(1-x^{*}\right)\left(1-y^{*}\right) \\
& -c^{2} s(1-s)(1-k)^{2}\left(1-x^{*}\right)\left(1-y^{*}\right)>0 .
\end{aligned}
$$

Hence, both eigenvalues have negative real part, and, hence, $\left(x^{*}, y^{*}\right)$ is locally asymptotically stable when $(1-k)<$ $(1-s) / s<1 /(1-k)$. Furthermore, when we mention that model (3) has no periodic orbits, refer to Lemma 3.2 of [16].

To summarize the above discussion, we give some sufficient conditions for the existence and asymptotical stability of equilibria for model (3).

Theorem 2. For any $k, s \in(0,1)$, one of the following statements is valid. (a) If $(1-s)<s(1-k)$, then model (3) has only two trivial equilibria $(1,0)$ and $(0,1)$, where $(0,1)$ is a saddle point and $(1,0)$ is globally asymptotically stable in the interior of $\Omega$.

(b) If $s<(1-s)(1-k)$, then model (3) has only two trivial equilibria $(1,0)$ and $(0,1)$, where $(1,0)$ is a saddle point and $(0,1)$ is globally asymptotically stable in the interior of $\Omega$.

(c) If $(1-k)<(1-s) / s<1 /(1-k)$, then model (3) has two saddle points $(1,0),(0,1)$ and a globally asymptotically stable positive equilibrium $\left(x^{*}, y^{*}\right)$.

Remark 3. From Theorem 2 and the equivalence of models (1) and (3), we obtain that model (1) has a globally asymptotically stable node $(1,0,0)$ and a saddle point $(0,1,0)$ for $(1-$ $s)<s(1-k)$ and has a saddle point $(1,0,0)$ and a globally asymptotically stable node $(0,1,0)$ for $s<(1-s)(1-k)$. Furthermore, if $(1-k)<(1-s) / s<1 /(1-k)$, model (1) has a coexistent equilibrium $\left(x^{*}, y^{*}, 1-x^{*}-y^{*}\right)$ which is globally asymptotically stable.

Finally, we fix all parameters including $c, k, s$, and $\alpha$ and carry out numerical investigations to confirm our main results obtained in this section. Firstly, we choose $k=0.5, s=$ $0.7, c=0.5$, and $\alpha=1$; it is easy to calculate that $1-$ $s=0.3<s(1-k)=0.7 \times 0.5=0.35$. So, from the first conclusion of Theorem 2, we know that model (1) has only two trivial equilibria $(1,0,0)$ and $(0,1,0),(1,0,0)$ is a globally asymptotically stable node, and $(0,1,0)$ is a saddle point, which is shown in Figure 1(a). It is clear that language $X$ is permanent and language $Y$ will fade away in this case. Similar conclusion can be obtained from Figure 1(b) with parameters $k=0.3, s=0.4, c=0.5$, and $\alpha=1$. However, if we choose $k=s=c=0.5$ and $\alpha=1$, then languages $X$ and $Y$ are coexistent and tend to positive equilibrium as shown in Figure 1(c).

\section{The Protection of an Endangered Language by a Continuous Control Strategy}

With the development of human civilization, people have taken effective measures to prevent the disappearance of language. In this section and the following, therefore, model (1) with parameters (2) is extended to assess the impact of control measures. And, in general, control strategies are divided into two main types: continuous control and pulse control. We, firstly, consider how a continuous control measure affects the dynamical behavior of model (1) in this section.

Through discussion of Theorem 2 in Section 2, we know that (i) language $X$ is permanent and language $Y$ and bilingual speakers $B$ are extinct for $(1-s)<s(1-k)$ and that (ii) language $Y$ is permanent and language $X$ and bilingual speakers $B$ are extinct for $s<(1-s)(1-k)$. Considering the similarities of the two cases, we only need to consider case (i); that is, $(1-s)<s(1-k)$.

3.1. Protecting of Language $Y$ with Continuous Control for $(1-s)<s(1-k)$. We consider model (1) with parameters (2) 


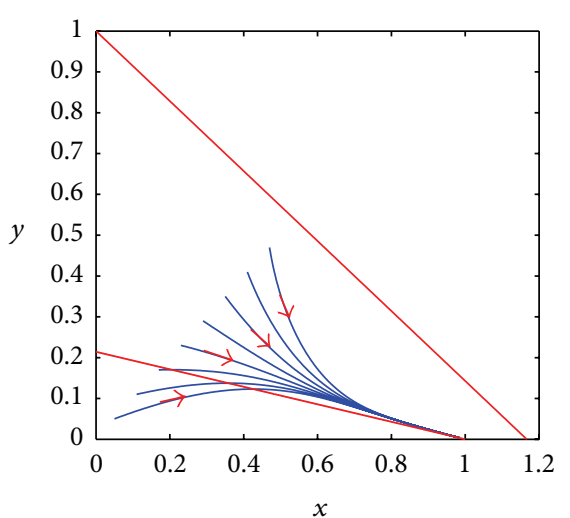

(a)

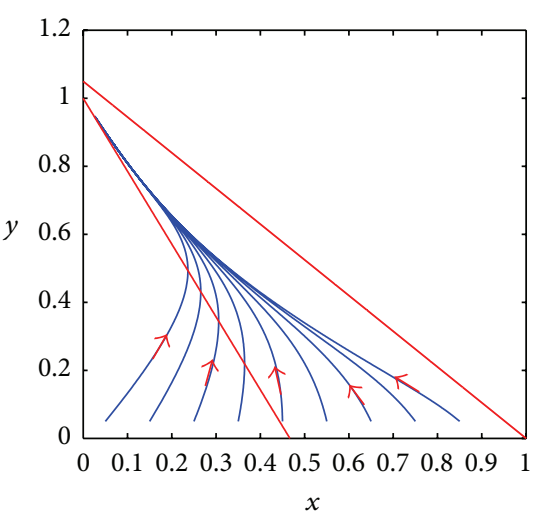

(b)

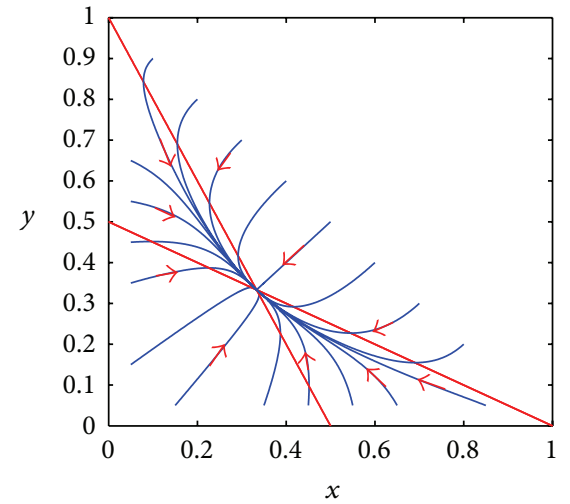

(c)

FIgURE 1: The asymptotical stability of equilibria of model (1) with $c=0.5$ and $\alpha=1$ : (a) the stability of trivial equilibrium $(1,0,0)$ with $k=$ 0.5 and $s=0.7$; (b) the stability of trivial equilibrium $(0,1,0)$ with $k=0.3$ and $s=0.7$; and (c) the stability of positive equilibrium $\left(x^{*}, y^{*}, 1-x^{*}-y^{*}\right)$ with $k=0.5$ and $s=0.5$.

and continuous control measure. The control system is modeled by the following differential equations:

$$
\begin{gathered}
\frac{\mathrm{d} x}{\mathrm{~d} t}=c s(1-k)(y+b)(1-y)-c(1-s) x(1-x)-c \mu x, \\
\frac{\mathrm{d} y}{\mathrm{~d} t}=c(1-s)(1-k)(x+b)(1-x)-c s y(1-y) \\
\frac{\mathrm{d} b}{\mathrm{~d} t}=c k(1-s) x(1-x)+c k s y(1-y) \\
\quad-c(1-k) b[(1-s)(1-x)+s(1-y)]+c \mu x
\end{gathered}
$$

where $\mu$ is a controlled variable, which means that the fraction of language $X$ becomes the bilingual $B$ per unit of time. Note that $x+y+b=1$; model (10) can be written as follows:

$$
\begin{gathered}
\frac{\mathrm{d} x}{\mathrm{~d} t}=c s(1-k)(1-x)(1-y)-c(1-s) x(1-x)-c \mu x \\
\frac{\mathrm{d} y}{\mathrm{~d} t}=c(1-s)(1-k)(1-y)(1-x)-c s y(1-y)
\end{gathered}
$$

We denote the right-side of model (11) by $f(x, y)$ and $g(x, y)$, respectively. We, here, discuss the existence and asymptotical stability of positive equilibrium of model (11).

From $g(x, y)=0$, it is easily shown that

$$
y_{\mu}=y(x)=\frac{(1-k)(1-s)(1-x)}{s} .
$$

Substituting it in $f(x, y)$, it follows that $f(1, y(1))=$ $-\mu<0$ and

$$
f(0, y(0))=c s(1-k)\left(1-\frac{(1-k)(1-s)}{s}\right)>0
$$

due to conditions $(1-s)<s(1-k)$ and $k, s \in(0,1)$. Hence, there is at least one $x_{\mu}^{*} \in(0,1)$ such that $f\left(x_{\mu}^{*}, y\left(x_{\mu}^{*}\right)\right)=0$. Furthermore, it is also easy to calculate that

$$
\begin{aligned}
& \frac{\mathrm{d} f(x, y(x))}{\mathrm{d} x}=c(1-k)[-s+2(1-k)(1-s)(1-x)] \\
& +c(1-s)(-1+2 x)-c \mu \\
& \frac{\mathrm{d}^{2} f(x, y(x))}{\mathrm{d} x^{2}}=2(1-s)\left[1-(1-k)^{2}\right]>0
\end{aligned}
$$

for all $x \in[0,1]$. This together with (14) gives that

$$
\frac{\mathrm{d} f(x, y(x))}{\mathrm{d} x}<\frac{\mathrm{d} f(1, y(1))}{\mathrm{d} x}=(1-s)-s(1-k)-\mu<0
$$

for all $x \in(0,1)$, where inequality $(1-s)<s(1-k)$ is used. Hence, $x_{\mu}^{*}$ is unique. That is, model (11) has a unique positive equilibrium $E_{\mu}\left(x_{\mu}^{*}, y_{\mu}^{*}\right)$ in the interior of $\Omega$.

Similar to the discussion of Theorem 2, we can get the locally asymptotical stability of equilibrium $E_{\mu}\left(x_{\mu}^{*}, y_{\mu}^{*}\right)$. Next, we discuss the global behavior of equilibrium $E_{\mu}\left(x_{\mu}^{*}, y_{\mu}^{*}\right)$. Let $B(x, y)=(1-x)^{-1}(1-y)^{-1}$; we have

$$
\frac{\partial(B f)}{\partial x}+\frac{\partial(B g)}{\partial y}=-\frac{c(1-s)}{1-y}-\frac{c s}{1-x}-\frac{c \mu}{(1-x)^{2}(1-y)}<0 .
$$

So model (11) has no closed orbit lying entirely in the interior of $\Omega$. We, therefore, can show from the above discussion that equilibrium $E_{\mu}\left(x_{\mu}^{*}, y_{\mu}^{*}\right)$ is globally asymptotically stable in the interior of $\Omega$. The result can be written by the following theorem.

Theorem 4. For any $\mu \in(0,1)$, if $(1-s)<s(1-k)$, then model (11) has a globally asymptotically stable positive equilibrium $E_{\mu}\left(x_{\mu}^{*}, y_{\mu}^{*}\right)$ in the interior of $\Omega$. 
Remark 5. From Theorem 2, it follows that language $X$ is permanent and that language $Y$ and bilingual speakers $B$ will eventually disappear for $(1-s)<s(1-k)$ in model (1). However, if we introduce a control variable $\mu$ (no matter how small it is) in model (1), languages $X$ and $Y$ are coexistent and tend to positive equilibrium $\left(x_{\mu}^{*}, y_{\mu}^{*}, 1-x_{\mu}^{*}-y_{\mu}^{*}\right)$. The coexistent state, of course, depends upon the controlled strength $\mu$. This implies that $\mu$ is a sensitive controlled parameter for the protection of endangered language.

3.2. Analysis of Optimal Control. Optimal control techniques are of great use in developing the optimal strategies to protect endangered civilization. To solve the challenges of obtaining an optimal control measure, we use optimal control theory; for more details, see Lenhart and Workman [27]. In model (10), for the optimal control problem, we consider a control variable $\mu(t) \in U_{a d}$; here, $U_{a d}=\{\mu: \mu(t)$ is measurable, $0 \leq$ $\mu(t) \leq 0.9$ for all $\left.t \in\left[0, t_{\text {final }}\right]\right\}$ indicates an admissible control. In this optimal problem, we assume a restriction on the control variable $\mu(t)$ such that $0 \leq \mu(t) \leq 0.9$, because conversion of all of language $X$ at one time is impossible. In case of no control, the fraction of language $X$ increases while the fractions of language $Y$ and bilingual $B$ die out. Therefore, the biological meaning of an optimal control in this problem is that the adequate levels for the fractions of language $X$ and bilingual $B$ are built.

Now, we consider an optimal control problem to maximize the objective functional

$$
J(\mu)=\int_{0}^{t_{\text {final }}}\left(C_{1} b-\frac{1}{2} C_{2} \mu^{2}\right) \mathrm{d} t
$$

subject to model (10). The first term represents the benefit of bilingual $B$, and the other term is systemic cost of control measure. The positive constants $C_{1}$ and $C_{2}$ balance the size of the terms $B$ and $\mu$. Our goal is maximizing the fraction of bilingual $B$ and minimizing the systemic cost to the control measure. This seeks an optimal control $\mu^{*}$ such that

$$
J\left(\mu^{*}\right)=\max \left\{J(\mu): \mu \in U_{a d}\right\}
$$

subject to model (10). It is obvious that the integrand of objective functional $J$ is a convex function of control variable $\mu$ and that state model satisfies the Lipschitz property with respect to the state since state solutions are bounded. The existence of an optimal control follows [28].

The necessary conditions that an optimal must satisfy come from the Pontryagin's Maximum Principle in [28]. This principle converts optimal control problem (10) and (18) into a problem of maximizing pointwise a Hamiltonian $H$ with respect to $\mu$ as follows:

$$
\begin{aligned}
& H= C_{1} b-\frac{1}{2} C_{2} \mu^{2}+\lambda_{1}[c s(1-k)(y+b)(1-y) \\
&-c(1-s) x(1-x)-c \mu x] \\
&+\lambda_{2}[c(1-s)(1-k)(x+b)(1-x)-c s y(1-y)]
\end{aligned}
$$

$$
\begin{gathered}
+\lambda_{3}\{c k(1-s) x(1-x)+c k s y(1-y)-c(1-k) b \\
\times[(1-s)(1-x)+s(1-y)]+c \mu x\}
\end{gathered}
$$

where $\lambda_{1}, \lambda_{2}$, and $\lambda_{3}$ are adjoint variables.

In the following theorem, we derive the necessary conditions for the optimal control problem.

Theorem 6. Let $\left(x^{*}, y^{*}, b^{*}\right)$ be an optimal state solution with associated optimal control variable $\mu^{*}$ for the maximized object functional $J(\mu)$ subject to control model (10). Then, for model (10), there exist adjoint variables $\lambda_{1}, \lambda_{2}$, and $\lambda_{3}$ such that

$$
\begin{aligned}
\frac{\mathrm{d} \lambda_{1}}{\mathrm{~d} t}= & \lambda_{1}\left[c(1-s)\left(1-x^{*}\right)-c(1-s) x^{*}+c \mu^{*}\right] \\
& +\lambda_{2}\left[c(1-s)(1-k)\left(x^{*}+b^{*}\right)-c(1-s)\right. \\
& \left.\quad \times(1-k)\left(1-x^{*}\right)\right] \\
& +\lambda_{3}\left[c k(1-s) x^{*}-c k(1-s)\left(1-x^{*}\right)\right. \\
& \left.-c(1-k)(1-s) b^{*}-c \mu^{*}\right], \\
\frac{\mathrm{d} \lambda_{2}}{\mathrm{~d} t}= & \lambda_{1}\left[c s(1-k)\left(y^{*}+b^{*}\right)-c s(1-k)\left(1-y^{*}\right)\right] \\
& +\lambda_{2}\left[c s\left(1-y^{*}\right)-c s y^{*}\right] \\
& +\lambda_{3}\left[c k s y^{*}-c k s\left(1-y^{*}\right)-c s(1-k) b^{*}\right], \\
\frac{\mathrm{d} \lambda_{3}}{\mathrm{~d} t}= & -C_{1}-\lambda_{1} c s(1-k)\left(1-y^{*}\right)-\lambda_{2} c(1-s) \\
& \times(1-k)\left(1-x^{*}\right) \\
& +\lambda_{3} c(1-k)\left[(1-s)\left(1-x^{*}\right)+s\left(1-y^{*}\right)\right]
\end{aligned}
$$

with transversality conditions $\lambda_{i}\left(t_{\text {final }}\right)=0, i=1,2,3$. Furthermore, the optimal control is given by

$$
\mu^{*}(t)=\min \left\{0.9, \max \left\{0, \frac{\left(\lambda_{3}-\lambda_{1}\right) c x^{*}}{A}\right\}\right\} .
$$

Proof. From Pontryagin's Maximum Principle, adjoint variables $\lambda_{i}(i=1,2,3)$ can be written as

$$
\frac{\mathrm{d} \lambda_{1}}{\mathrm{~d} t}=-\frac{\partial H}{\partial x}, \quad \frac{\mathrm{d} \lambda_{2}}{\mathrm{~d} t}=-\frac{\partial H}{\partial y}, \quad \frac{\mathrm{d} \lambda_{3}}{\mathrm{~d} t}=-\frac{\partial H}{\partial b}
$$

These are just differential equations (21)-(23) with transversality conditions $\lambda_{i}\left(t_{\text {final }}\right)=0(i=1,2,3)$. Furthermore, by optimality condition, we have that

$$
\left.\frac{\partial H}{\partial \mu}\right|_{\mu=\mu^{*}}=-C_{2} \mu^{*}-c \lambda_{1} x^{*}+c \lambda_{3} x^{*}=0
$$

This shows that

$$
\mu^{*}=\frac{\left(\lambda_{3}-\lambda_{1}\right) c x^{*}}{C_{2}} .
$$


Using the property of control space, we get that

$$
\mu^{*}= \begin{cases}0, & \frac{\left(\lambda_{3}-\lambda_{1}\right) c x^{*}}{C_{2}} \leq 0, \\ \frac{\left(\lambda_{3}-\lambda_{1}\right) c x^{*}}{C_{2}}, & 0<\frac{\left(\lambda_{3}-\lambda_{1}\right) c x^{*}}{C_{2}}<0.9 \\ 0.9, & \frac{\left(\lambda_{3}-\lambda_{1}\right) c x^{*}}{C_{2}} \geq 0.9 .\end{cases}
$$

This can be rewritten in compact notation, which is just (24). This completes the proof.

3.3. Numerical Simulation and Discussion. Here, we discuss how the continuous control measure affects the protection of endangered language and the existence and stability of positive equilibrium for model (10) by numerical simulations. Firstly, we choose the same parameters as in Figure 1(a); that is, $c=k=0.5$ and $s=0.7$. Besides that, we choose control variable $\mu=0.25$. Figure 2(a), however, is completely different from Figure 1(a), which shows that model (10) has a globally asymptotically stable positive equilibrium $\left(x_{\mu}^{*}, y_{\mu}^{*}, b_{\mu}^{*}\right)=(0.4194,0.1244,0.4562)$. Furthermore, from the discussion of Theorem 4 , it follows that values $x_{\mu}^{*}$ and $y_{\mu}^{*}$ will decrease and increase with increase in control strength $\mu$, respectively, which is shown in Figures 2(b) and 2(c). The strong consistency between theoretical result and real situation is obviously observed.

In addition, in Figure 2(c), it is interesting to note that control strength $\mu$ is close to 1 (where $\mu=0.95$ ), but language $X$ runs around 0.2 . This means that, no matter how strong the control strength $\mu$ is, language $X$ will not fade. Namely, control measure can only protect endangered language $Y$, but not result in extinction of language $X$. Actually, in the real world, the own characteristics are very important factors in determining the development of languages.

The plots in Figure 3(a) show three adjoint variables $\lambda_{1}, \lambda_{2}$, and $\lambda_{3}$ in the optimality system. We solve these adjoint equations by a backward Runge-Kutta fourth-order procedure because of the transversality conditions for more details, see Lenhart and Workman [27]. In Figure 3(b), dotted line and solid line represent languages $X, Y$ and bilingual $B$ in model (10) without and with continuous control, respectively. We see that the fractions of language $Y$ and bilingual $B$ in population decrease more when there is no control. In this case, most of this population goes to language $X$. If we apply the continuous control measure, however, the fraction of language $Y$ slowly falls, the fraction of language $X$ decreases quite a lot, and the fraction of bilingual $B$ is quite greater than the fraction in the case without control, since our main object is maximizing the fraction of bilingual $B$. In Figure $3(\mathrm{c})$, the control $\mu$ is plotted as a function of time for three different values of weight factor $C_{2}: 0.045,0.1$, and 0.5 . The control variable $\mu$ for the associated weight factor $\mu=$ 0.045 is much larger than the other two values. Note that, in general, as $C_{2}$ decreases, the amount of $\mu$ increases. The same results can also be obtained from the expression of $\mu$ in (27). The associated weight factor $C_{2}$ also plays a significant role in keeping the balance of the size of fraction an optimal problem.

\section{Protection of Language $Y$ with Impulsive Control for $(1-s)<s(1-k)$}

For reasons of protecting culture diversity, in this section, we will consider how the state-dependent pulse control measure affects the prevention of endangered language. Since learning cycle is very brief in contrast to the life cycle of a person, naturally we suppose that the procedure of learning is pulse effect.

As for the protection of endangered languages for the state-dependent control measure, we construct the following controlled model which is modeled by differential equations with state-dependent pulse effect:

$$
\begin{aligned}
& \frac{\mathrm{d} x}{\mathrm{~d} t}=y P_{Y X}+b P_{B X}-x\left(P_{X Y}+P_{X B}\right), \\
& \frac{\mathrm{d} y}{\mathrm{~d} t}=x P_{X Y}+b P_{B Y}-y\left(P_{Y X}+P_{Y B}\right), \\
& \frac{\mathrm{d} b}{\mathrm{~d} t}=x P_{X B}+y P_{Y B}-b\left(P_{B Y}+P_{B X}\right), \\
& x\left(t^{+}\right)=(1-\theta) x(t), \\
& y\left(t^{+}\right)=y(t), \\
& b\left(t^{+}\right)=b(t)+\theta x(t), \\
& x=E_{X} .
\end{aligned}
$$

The meaning of model (29) is as follows: when the fraction of language $X$ reaches the critical threshold value $E_{X}$ at time $t_{E}$, controlling measure (for example, encouraging some speaker of language $X$ to study language $Y$ ) is taken and the fractions of language $X$ and bilingual speakers $B$ immediately become $(1-\theta) x\left(t_{E}\right)$ and $b\left(t_{E}\right)+\theta x\left(t_{E}\right)$, respectively.

Remark 7. It is obvious that the fractions of language $Y$ and bilingual $B$ are rather small and in danger of becoming extinct when the fraction of language $X$ reaches the critical threshold value $E_{X}$. In this case, the effective measure is taken to prevent the loss of language $Y$. The times of control measures are obviously related to the state of language $X$.

Remark 8. The critical threshold value $E_{X}$ represents the fraction of monolingual speakers $X$ in population, and $\theta$ represents the strength of control measure. Numerical simulations in Section 4.3 show that these are crucial parameters in model (29).

For model (29), which is equivalent to the following reduced model since the population has a constant size $x+$ $y+b=1$,

$$
\begin{array}{r}
\frac{\mathrm{d} x}{\mathrm{~d} t}=c(1-x)[(1-k) s(x+b)-x(1-s)], \\
\frac{\mathrm{d} b}{\mathrm{~d} t}=c(1-x)[k x-b(1-s-k)]-b c s(x+b), \\
x \neq E_{X},
\end{array}
$$




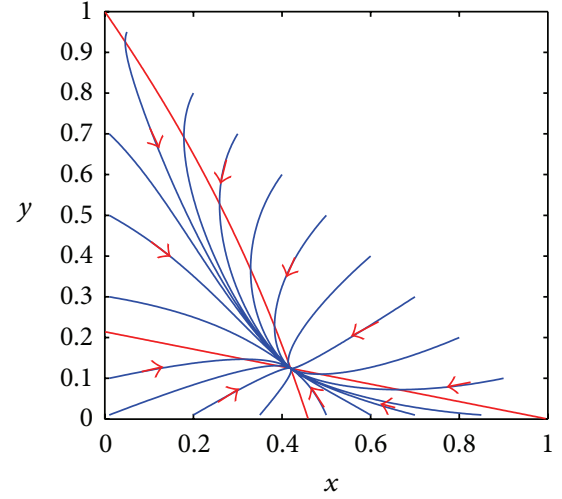

(a)

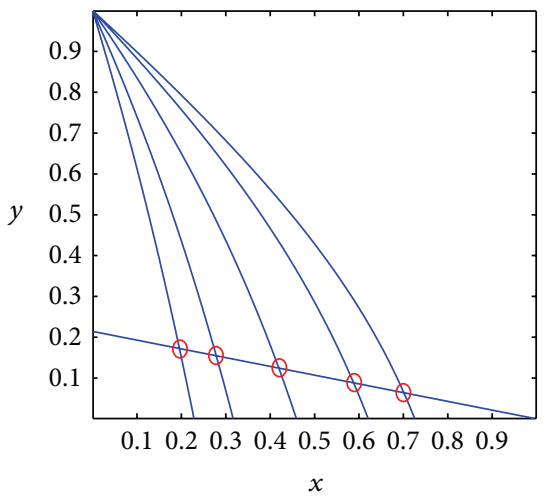

(b)

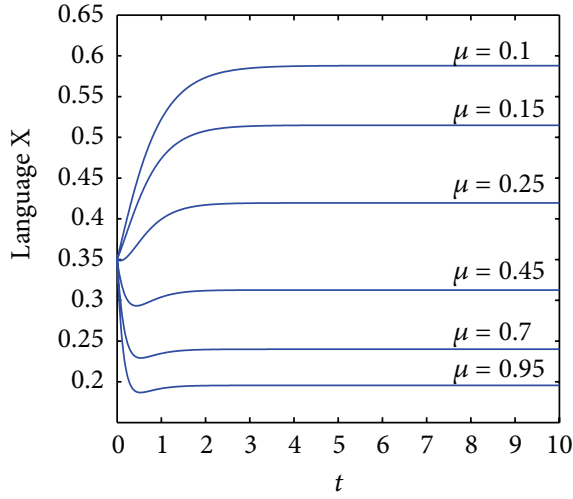

(c)

FIGURE 2: The effect of control measure on the existence and stability of positive equilibrium for model (10) with $c=k=0.5, s=0.7$, and $\alpha=1$.

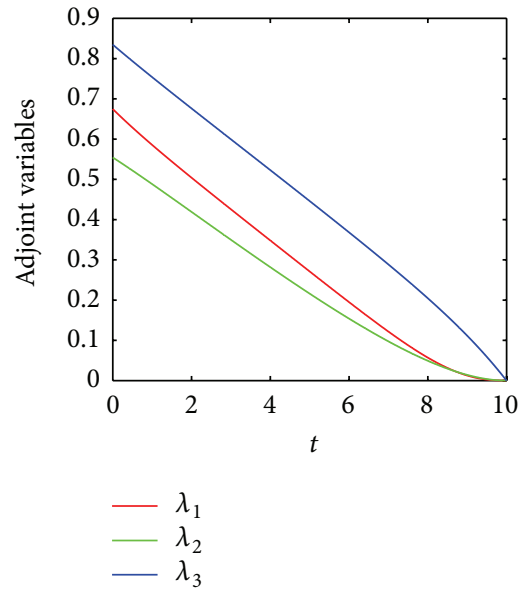

(a)

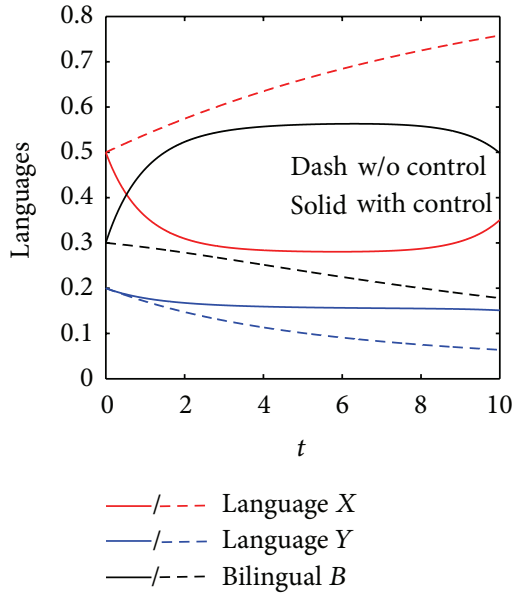

(b)

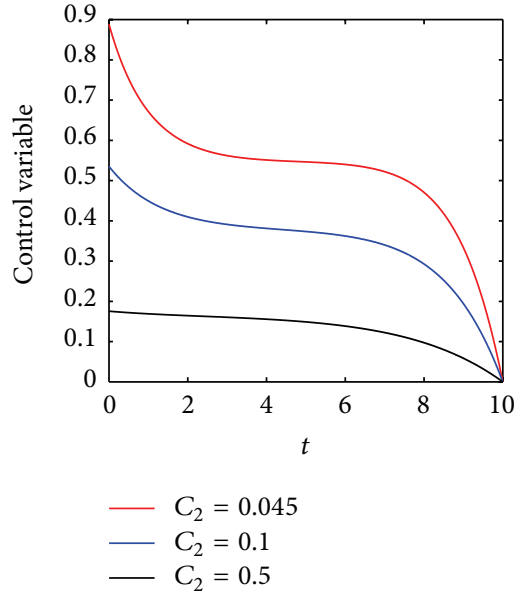

(c)

Figure 3: The optimal adjoint variables, states, and control variable for the optimal control problem with $c=k=0.5, s=0.7, \alpha=1$, and $C_{1}=0.1$ : (a) the optimal adjoint variables $\lambda_{1}, \lambda_{2}$, and $\lambda_{3}$; (b) the optimal states of languages $X, Y$ and bilingual $B$; and (c) the control variable $\mu$ with different weight factor $C_{2}$.

$$
\begin{array}{r}
x\left(t^{+}\right)=(1-\theta) x(t), \\
b\left(t^{+}\right)=b(t)+\theta x(t), \\
x=E_{X} .
\end{array}
$$

By the biological background of model (30), we only consider model in the biological meaning region $\Omega_{0}=$ $\{(x, b): x \geq 0, b \geq 0, x+b \leq 1\}$. Obviously, the global existence and uniqueness of solution of model (30) are guaranteed by the smoothness properties of the right-side of model (30); for more details, see Lakshmikantham et al. [29].

4.1. Preliminaries. To discuss the dynamic behavior of model (30), we define two cross-sections to the phase space of model (30) by sections

$$
\begin{gathered}
\Gamma_{\theta}=\left\{(x, b): x=(1-\theta) E_{X}, b \geq 0\right\}, \\
\Gamma_{E}=\left\{(x, b): x=E_{X}, b \geq 0\right\} .
\end{gathered}
$$

Suppose that point $A_{n}\left(E_{X}, b_{n}\right)$ is on section $\Gamma_{E}$; then, trajectory

$$
\begin{array}{r}
O^{+}\left(A_{n}, t_{n}\right)=\left\{(x(t), b(t)):(x(t), b(t)) \in \Omega_{0},\right. \\
\left.t \geq t_{n}, x\left(t_{n}\right)=E_{X}, b\left(t_{n}\right)=b_{n}\right\}
\end{array}
$$

jumps to point $A_{n}^{+}\left((1-\theta) E_{X}, \widehat{b}_{n}\right)$ on section $\Gamma_{\theta}$ due to pulse effects $x\left(t_{n}^{+}\right)=(1-m) x\left(t_{n}\right)$ and $b\left(t_{n}^{+}\right)=b\left(t_{n}\right)+\theta x\left(t_{n}\right)$, then intersects section $\Gamma_{E}$ at point $A_{n+1}\left(E_{X}, b_{n+1}\right)$, and finally jumps to point $A_{n+1}^{+}\left((1-\theta) E_{X}, \widehat{b}_{n+1}\right)$ on section $\Gamma_{\theta}$ again, where $b_{n+1}$ is decided by $b_{n}$ and parameters $\theta, E_{X}$. Therefore, we defined a Poincaré map of section $\Gamma_{E}$ as follows:

$$
b_{n+1}=F\left(b_{n}, \theta, E_{X}\right) .
$$

Let $z(t)=(x(t), b(t))$ be a solution of model (30) with initial condition $z_{0}=z\left(t_{0}\right)=\left((1-\theta) E_{X}, \widehat{b}_{0}\right)$. Trajectory $\mathrm{O}^{+}\left(z_{0}, t_{0}\right)$ starts from point $A_{0}\left((1-\theta) E_{X}, \widehat{b}_{0}\right)$; first, it intersects section $\Gamma_{E}$ at point $B_{1}\left(E_{X}, b_{1}\right)$, then jumps to point 
$A_{1}\left((1-\theta) E_{X}, \widehat{b}_{1}\right)$ on section $\Gamma_{\theta}$ due to pulse effects, and finally reaches point $B_{2}\left(E_{x}, b_{2}\right)$ on section $\Gamma_{E}$ again. Repeating the above process, we have two points' sequences $\left\{A_{n-1}\left((1-\theta) E_{X}, \widehat{b}_{n-1}\right)\right\}$ and $\left\{B_{n}\left(E_{X}, b_{n}\right)\right\}(n=1,2, \ldots)$. We notice that the coordinates satisfy the relation $\widehat{b}_{n}=b_{n}+$ $\theta E_{X}(n=1,2, \ldots)$.

Let $\mathbb{S}$ be an arbitrary set in $\mathbb{R}^{2}$, and let $P$ be an arbitrary point in $\mathbb{R}^{2}$. The distance between point $P$ and set $\mathbb{S}$ is defined by $d(P, \mathbb{S})=\inf _{P_{0} \in \mathbb{S}}\left|P-P_{0}\right|$. For the convenience of statement in the rest of this paper, we introduce some definitions.

Definition 9. Trajectory $\mathrm{O}^{+}\left(z_{0}, t_{0}\right)$ of model (30) is said to be order- $k$ periodic if there exists a positive integer $k \geq 1$ such that $k$ is the smallest integer for $\widehat{b}_{0}=\widehat{b}_{k}$.

Definition 10 (orbital stability [30]). Trajectory $\mathrm{O}^{+}\left(X_{0}, t_{0}\right)$ is said to be orbitally stable if, given that $\varepsilon>0$, there exists a constant $\delta=\delta(\varepsilon)>0$ such that, for other solution $z^{*}(t)$ of model (30), when $d\left(z^{*}\left(t_{0}\right), O^{+}\left(z_{0}, t_{0}\right)\right)<\delta$, one has that $d\left(z^{*}(t), O^{+}\left(z_{0}, t_{0}\right)\right)<\varepsilon$ for all $t>t_{0}$.

Definition 11 (orbitally asymptotical stability [30]). Trajectory $\mathrm{O}^{+}\left(z_{0}, t_{0}\right)$ is said to be orbitally asymptotically stable if it is orbitally stable, and there exists a constant $\eta>0$ such that, for any other solution $z^{*}(t)$ of model (30), when $d\left(z^{*}\left(t_{0}\right), O^{+}\left(z_{0}, t_{0}\right)\right)<\eta, \lim _{t \rightarrow \infty} d\left(z^{*}(t), O^{+}\left(z_{0}, t_{0}\right)\right)=0$.

Next, we consider the following autonomous model with pulse effects:

$$
\begin{array}{llll}
\frac{\mathrm{d} x}{\mathrm{~d} t} & =f(x, y), & \frac{\mathrm{d} y}{\mathrm{~d} t}=g(x, y), & \varphi(x, y) \neq 0, \\
\Delta x & =\xi(x, y), & \Delta y=\eta(x, y), & \varphi(x, y)=0,
\end{array}
$$

where $f(x, y)$ and $g(x, y)$ are continuous differential functions defined on $\mathbb{R}^{2}$ and $\varphi(x, y)$ is a sufficiently smooth function with $\nabla \varphi(x, y) \neq 0$. Let $(\mu(t), \nu(t))$ be a positive $T$ periodic solution of model (34). The following result comes from Corollary 2 of Theorem 1 in [31].

Lemma 12 (analogue of Poincaré criterion). If the Floquet multiplier $\mu$ satisfies condition $|\mu|<1$, where

$$
\mu=\prod_{j=1}^{n} \kappa_{j} \exp \left\{\int_{0}^{T}\left[\frac{\partial f(\mu(t), \nu(t))}{\partial x}+\frac{\partial g(\mu(t), \nu(t))}{\partial y}\right] \mathrm{d} t\right\}
$$

with

$$
\begin{gathered}
\kappa_{j}=\left(\left(\frac{\partial \eta}{\partial y} \frac{\partial \varphi}{\partial x}-\frac{\partial \eta}{\partial x} \frac{\partial \varphi}{\partial y}+\frac{\partial \varphi}{\partial x}\right) f_{+}\right. \\
+\left(\frac{\partial \xi}{\partial x} \frac{\partial \varphi}{\partial y}-\frac{\partial \xi}{\partial y} \frac{\partial \varphi}{\partial x}\right. \\
\left.\left.+\frac{\partial \varphi}{\partial y}\right) g_{+}\right) \\
\times\left(\frac{\partial \varphi}{\partial x} f+\frac{\partial \varphi}{\partial y} g\right)^{-1}
\end{gathered}
$$

and $f, g, \partial \xi / \partial x, \partial \xi / \partial y, \partial \eta / \partial x, \partial \eta / \partial y, \partial \varphi / \partial x$, and $\partial \varphi / \partial y$ have been calculated at the point $\left(\mu\left(\tau_{j}\right), \nu\left(\tau_{j}\right)\right), f_{+}=$ $f\left(\mu\left(\tau_{j}^{+}\right), v\left(\tau_{j}^{+}\right)\right), g_{+}=g\left(\mu\left(\tau_{j}^{+}\right), v\left(\tau_{j}^{+}\right)\right)$, and $\tau_{j}(j \in N)$ is the time of the jth jump, then, $(\mu(t), \nu(t))$ is orbitally asymptotically stable.

4.2. Main Results. On the existence of positive order-1 periodic solution for model (30), we have the following theorem.

Theorem 13. For any $\theta \in(0,1)$ and $E_{X} \in(0,1)$, model (30) admits a positive order-1 periodic solution.

Proof. Let point $A_{0}\left((1-\theta) E_{X}, \widehat{s}_{0}\right) \in \Gamma_{\theta}$ for sufficiently small $\widehat{s}_{0}$ with $\widehat{s}_{0} \leq \theta E_{X}$. In view of the geometrical structure of the phase space of model (30), trajectory $\mathrm{O}^{+}\left(A_{0}, t_{0}\right)$ of model (30) starts from initial point $A_{0}$ and intersects section $\Gamma_{E}$ at point $B_{1}\left(E_{X}, s_{1}\right)$. And then, trajectory $O^{+}\left(A_{0}, t_{0}\right)$ jumps to point $A_{1}\left(\widehat{s}_{1},(1-\theta) E_{X}\right)$ on section $\Gamma_{\theta}$ due to pulse control effects $x\left(t^{+}\right)=(1-\theta) x(t)$ and $b\left(t^{+}\right)=b(t)+\theta x(t)$. Since $\widehat{s}_{0} \leq \theta E_{X}$, it follows that point $A_{1}$ is above point $A_{0}$. Furthermore, point $B_{2}$ is above point $B_{1}$. Otherwise, this is a contradiction with the uniqueness of solution for model (30). By (33), we have that $s_{2}=F\left(s_{1}, \theta, E_{X}\right)$ and

$$
F\left(s_{1}, \theta, E_{X}\right)-s_{1}=s_{2}-s_{1}>0
$$

On the other hand, let $\lambda_{0}=1-(1-\theta) E_{X}$. For critical point $C_{0}\left((1-\theta) E_{X}, \lambda_{0}\right)$, trajectory $O^{+}\left(C_{0}, t_{0}\right)$ starts from initial point $C_{0}$; it intersects section $\Gamma_{E}$ at point $D_{1}\left(E_{X}, \lambda_{1}\right)$, then jumps to point $C_{1}\left((1-m) E_{X}, \lambda_{1}^{+}\right)$on section $\Gamma_{\theta}$, and finally reaches point $D_{2}\left(E_{X}, \lambda_{2}\right)$ on section $\Gamma_{E}$ again. Since $x+b \leq$ 1 , one has that $(1-m) E_{X}+\lambda_{1}^{+} \leq 1=(1-\theta) E_{X}+\lambda_{0}$. That is, $\lambda_{1}^{+} \leq \lambda_{0}$. If $\lambda_{1}^{+}=\lambda_{0}$, namely, points $C_{0}$ and $C_{1}$ coincide, then model (30) has a positive order-1 periodic solution. Otherwise, $\lambda_{1}^{+}<\lambda_{0}$; that is, point $C_{0}$ is above point $C_{1}$, and point $D_{1}$ is above point $D_{2}$. By (33), we can get that $\lambda_{2}=$ $F\left(\lambda_{1}, \theta, E_{X}\right)$ and

$$
F\left(\lambda_{1}, \theta, E_{X}\right)-\lambda_{1}=\lambda_{2}-\lambda_{1}<0 \text {. }
$$

This together with (37) yields that Poincaré map (33) has a fixed point; that is, model (30) has a positive order-1 periodic solution. This completes the proof.

Now, on the orbital stability of positive order-1 periodic solution of model (30), we have the following result.

Theorem 14. Let $(\varphi(t), \psi(t))$ be a positive order-1 periodic solution of model $(30)$ with period $T$. For any $\theta \in(0,1)$ and $E_{X} \in(0,1)$, if the Floquet multiplier $\mu$ satisfies condition $|\mu|<$ 1 , where

$$
\begin{array}{r}
|\mu|=|\kappa| \exp \left\{\int_{0}^{T}[c(1-\varphi(t))(3 s+k-k s-2)\right. \\
-c s(\varphi(t)+\psi(t))-c s \psi(t)] \mathrm{d} t\}
\end{array}
$$


with

$$
\kappa=\frac{(1-k) s\left(E_{X}+\psi(T)\right)-(1-\theta) E_{X}(1-s)}{(1-k) s\left(E_{X}+\psi(T)\right)-E_{X}(1-s)},
$$

then $(\varphi(t), \psi(t))$ is orbitally asymptotically stable.

Proof. Suppose that $(\varphi(t), \psi(t))$ intersects sections $\Gamma_{\theta}$ and $\Gamma_{E}$ at points $C^{+}\left((1-\theta) E_{X}, \theta E_{X}+\psi(T)\right)$ and $C\left(E_{X}, \psi(T)\right)$, respectively. Comparing with model (34), we have that

$$
\begin{gathered}
f(x, b)=c(1-x)[(1-k) s(x+b)-x(1-s)], \\
g(x, b)=c(1-x)[k x-b(1-s-k)]-b c s(x+b),
\end{gathered}
$$

and $\xi(x, b)=-\theta x, \eta(x, b)=\theta x, \phi(S, I)=x-E_{X}$, $(\varphi(T), \psi(T))=\left(E_{X}, \psi(T)\right)$, and $\left(\varphi\left(T^{+}\right), \psi\left(T^{+}\right)\right)=((1-$ $\left.\theta) E_{X}, \psi(T)+\theta E_{X}\right)$. Thus,

$$
\begin{gathered}
\frac{\partial f}{\partial x}=-c[(1-k) s(x+b)-x(1-s)] \\
+c(1-x)(2 s-k s-1), \\
\frac{\partial g}{\partial b}=-c(1-x)(1-s-k)-c s(x+b)-c s b, \\
\frac{\partial \xi}{\partial x}=-\theta, \quad \frac{\partial \eta}{\partial x}=\theta, \\
\frac{\partial \phi}{\partial x}=1, \quad \frac{\partial \xi}{\partial b}=\frac{\partial \eta}{\partial b}=\frac{\partial \phi}{\partial b}=0 .
\end{gathered}
$$

Furthermore, it follows from (42) that

$$
\begin{aligned}
\kappa= & \left(\left(\frac{\partial \eta}{\partial b} \frac{\partial \phi}{\partial x}-\frac{\partial \eta}{\partial x} \frac{\partial \phi}{\partial b}+\frac{\partial \phi}{\partial x}\right) f_{+}\right. \\
& \left.+\left(\frac{\partial \xi}{\partial x} \frac{\partial \phi}{\partial b}-\frac{\partial \xi}{\partial b} \frac{\partial \phi}{\partial x}+\frac{\partial \phi}{\partial b}\right) g_{+}\right) \times\left(\frac{\partial \phi}{\partial x} f+\frac{\partial \phi}{\partial b} g\right)^{-1} \\
= & \frac{f_{+}\left(\varphi\left(T^{+}\right), \psi\left(T^{+}\right)\right)}{f(\varphi(T), \psi(T))} \\
= & \left(( 1 - ( 1 - \theta ) E _ { X } ) \left[(1-k) s\left(E_{X}+\psi(T)\right)\right.\right. \\
& \times\left(( 1 - E _ { X } ) \left[( 1 - k ) s \left(E_{X}\right.\right.\right. \\
& \left.\left.+\psi(T))-E_{X}(1-s)\right]\right)^{-1},
\end{aligned}
$$$$
\mu=\kappa \exp \left\{\int_{0}^{T}[-c((1-k) s(\varphi(t)+\psi(t))\right.
$$$$
-\varphi(t)(1-s))+c(1-\varphi(t))
$$$$
\times(2 s-k s-1)-c(1-\varphi(t))(1-s-k)
$$$$
-c s(\varphi(t)+\psi(t))-c s \psi(t)] \mathrm{d} t\} .
$$

On the other hand, integrating both sides of the first equation of model (30) along the orbit $\widehat{C^{+} C}$, we have that

$$
\begin{aligned}
\ln \frac{1-E_{X}}{1-(1-\theta) E_{X}} & =\int_{(1-\theta) E_{X}}^{E_{X}} \frac{\mathrm{d} x}{1-x} \\
& =-\int_{0}^{T} c[(1-k) s(\varphi(t)+\psi(t)) \\
& -\varphi(t)(1-s)] \mathrm{d} t .
\end{aligned}
$$

From (43)-(45), we can obtain that

$$
\begin{aligned}
|\mu|=\left|\frac{(1-k) s\left(E_{X}+\psi(T)\right)-(1-\theta) E_{X}(1-s)}{(1-k) s\left(E_{X}+\psi(T)\right)-E_{X}(1-s)}\right| & \quad \times \exp \left\{\int_{0}^{T}[c(1-\varphi(t))(3 s+k-k s-2)\right. \\
& -c s(\varphi(t)+\psi(t))-c s \psi(t)] \mathrm{d} t\} .
\end{aligned}
$$

By condition (39), we see that model (30) satisfies all conditions of Lemma 12. Therefore, order-1 periodic solution $(\varphi(t), \psi(t))$ of model (30) is orbitally asymptotically stable and has asymptotic phase property. This completes the proof.

Remark 15. From Theorem 14, though condition (39) of Theorem 14 is hard to test, yet it is weak since the second and third items of the exponent term of the right-side of (39) are negative.

Next, we give a more general result on the existence and stability of positive order-1 periodic solutions of model (30).

Theorem 16. For any $\theta \in(0,1)$ and $E_{X} \in(0,1)$, model (30) has a positive order-1 periodic solution which is orbitally asymptotically stable.

Proof. Let $\lambda^{*}=1-(1-\theta) E_{X}$, and suppose that the trajectory $\mathrm{O}^{+}\left(C_{0}, t_{0}\right)$ of model (30) starts from critical point $C_{0}((1-$ $\left.\theta) E_{X}, \lambda^{*}\right)$ and intersects section $\Gamma_{E}$ at point $C^{*}\left(E_{X}, \beta^{*}\right)$. In view of the geometrical construction of phase space of model $(30)$ and that $(1,0)$ is a globally asymptotically stable node, we obtain that trajectory of model (30) which starts from point $\left((1-\theta) E_{X}, b\right)$ with $b \in\left(0, \lambda^{*}\right)$ will intersect section $\Gamma_{E}$ at point $\left(E_{X}, \widehat{b}\right)$; then, $\widehat{b} \in\left(0, \beta^{*}\right)$. So we only need to consider trajectories of model (30) which start from the point $\left(E_{X}, b\right)$ on section $\Gamma_{E}$, where $b \in\left(0, \beta^{*}\right)$.

Suppose that trajectory $\mathrm{O}^{+}\left(D_{1}, t_{0}\right)$ of model (30) which starts from initial point $D_{1}\left(E_{X}, b_{1}\right)\left(0<b_{1}<\beta^{*}\right)$ jumps to point $D_{1}^{+}\left((1-\theta) E_{X}, \widehat{b}_{1}\right)$ on section $\Gamma_{\theta}$ due to pulse effects and then reaches section $\Gamma_{E}$ at point $D_{2}\left(E_{X}, b_{2}\right)$, where $\hat{b}_{1} \in\left(0, \lambda^{*}\right)$ and $b_{2} \in\left(0, \beta^{*}\right)$. Repeating the above process, we have pulse points sequences $\left\{D_{n}\left(E_{X}, b_{n}\right)\right\}$ and $\left\{D_{n}^{+}\left((1-\theta) E_{X}, \widehat{b}_{n}\right)\right\}$, where $b_{n} \in\left(0, \beta^{*}\right)$ and $\widehat{b}_{n} \epsilon$ $\left(0, \lambda^{*}\right)$. Furthermore, this follows from Poincaré map (33) that $b_{n+1}=F\left(b_{n}, \theta, E_{X}\right)(n=1,2, \ldots)$. On the other hand, for any two points $D_{i}\left(E_{X}, b_{i}\right)$ and $D_{j}\left(E_{X}, b_{j}\right)$ on section $\Gamma_{E}$, where $b_{i}, b_{j} \in\left(0, \beta^{*}\right)$ and $b_{i}<b_{j}$, in view of pulse effects, 
point $D_{i}^{+}\left((1-\theta) E_{X}, \widehat{b}_{i}\right)$ is below point $D_{j}^{+}\left((1-\theta) E_{X}, \widehat{b}_{j}\right)$. And then, trajectories $O^{+}\left(D_{i}, t_{0}\right)$ and $O^{+}\left(D_{j}, t_{0}\right)$ intersect section $\Gamma_{E}$ at points $D_{i+1}\left(E_{X}, b_{i+1}\right)$ and $D_{j+1}\left(E_{X}, b_{j+1}\right)$, respectively. Therefore, from the geometrical construction of the phase space of model (30), we claim that

$$
0<b_{i+1}<b_{j+1}<\beta^{*} .
$$

In fact, if inequality (47) does not hold, that is, $b_{i+1} \geq b_{j+1}$, then, it follows that point $D_{i+1}$ is above point $D_{j+1}$ or that two points coincide. Furthermore, we can obtain that trajectories $\mathrm{O}^{+}\left(D_{i}, t_{0}\right)$ and $O^{+}\left(D_{j}, t_{0}\right)$ intersect at a point $D^{*}(\widehat{x}, \widehat{b})$. It is indicated that there are two different solutions which start from point $D^{*}$. This is a contradiction with the uniqueness of solution of model (30). So inequality (47) is thus valid.

Now, for any $b_{0} \in\left(0, \beta^{*}\right)$, from Poincaré map (33) of section $\Gamma_{E}$, we have $b_{1}=F\left(b_{0}, \theta, E_{X}\right), b_{2}=F\left(b_{1}, \theta, E_{X}\right)$, and $b_{n+1}=F\left(b_{n}, \theta, E_{X}\right)(n=2,3,4, \ldots)$. In particular, if $b_{0}=b_{1}$, then model (30) has a positive order-1 periodic solution, if $b_{0} \neq b_{1}$, then it follows from the fact (47) that $b_{0} \neq b_{2}$.

Next, we discuss the general circumstance; that is, $b_{0} \neq b_{1} \neq b_{2} \neq \cdots \neq b_{k}(k>2)$.

(a) If $b_{0}<b_{1}$, from (47), we obtain that $b_{1}<b_{2}$. Then, $b_{0}<b_{1}<b_{2}$. In this case, repeating the above process, we have that

$$
0<b_{0}<b_{1}<b_{2}<b_{3} \cdots<\beta^{*} .
$$

(b) If $b_{0}>b_{1}$, similar to (a), we have that

$$
0<\cdots<b_{3}<b_{2}<b_{1}<b_{0}<\beta^{*} .
$$

Therefore, in case (a), we can get that $\lim _{n \rightarrow \infty} b_{n}=\eta$. This implies that model (30) has an orbitally asymptotically stable positive order-1 periodic solution. Similarly, we can obtain the same result in case (b). This completes the proof.

Remark 17. According to the equivalence of models (29) and (30), from Theorem 16, we also obtained that model (29) has a positive order-1 periodic solution which is orbitally asymptotically stable. At the same time, it also implies that languages $X$ and $Y$ are coexistent and have a stable equilibrium state under state-dependent impulsive control strategy.

Remark 18. Similar results can also be obtained from the case that $s<(1-s)(1-k)$; we, hence, omit them here.

4.3. Numerical Simulation and Discussion. In this subsection, some numerical simulations are carried out to illustrate main results and the feasibility of state-dependent pulse feedback control measure. Firstly, we choose model parameters $c=$ $k=0.5$ and $s=0.7$ and control parameters $E_{X}=0.8$ and $\theta=0.15$. From Figure 4, we see that state-dependent control measure plays an important role in preventing the disappearance of endangered language. Under statedependent impulsive control measure, the downward trend for language $Y$ was controlled effectively, and the fractions of language $X$ and bilingual $B$ are kept within reasonable levels. Furthermore, numerical simulations also show that the fractions of languages $X, Y$ and bilingual $B$, though from different initial states, are stabilized in the same state. The corresponding numerical results are presented in Figures 4 and 5. Namely, model (29) has a positive order-1 periodic solution, which is orbitally asymptotically stable. This is certainly the case as shown in Theorems 13-16. Again from Figure 5(a)$5(\mathrm{c})$, the periodic solution is orbitally asymptotically stable instead of being Lyapunov asymptotically stable. In fact, it also shows exactly how different the two stabilities are.

Next, we investigate what effect has the choice of controlling parameters on the dynamical behavior of model (29) using numerical modeling method. We first choose $E_{X}=$ 0.8 and parameter $\theta$ to be $0.05,0.15,0.25$, and 0.35 , respectively. From Figure 6, we note that the length of times intervals between two control strategies are closely geared to the strength of control measure $\theta$ and that the time interval increases with the increasing of $\theta$. Again from Figures 6(b) and 6(c), it is obvious that the fractions of language $Y$ and bilingual $B$ in population could maintain higher level for a long time due to larger $\theta$. Of course, the cost of control measure is related to its strength. Furthermore, similar results can also be obtained from $\theta=0.15$ and letting $E_{X}$ be $0.9,0.85,0.8$, and 0.7 in Figure 7. It is not hard to imagine, however, that the cost of control measure is very high if the fraction of monolingual speakers is incredibly low in the population. This is because if the fraction of monolingual speakers is high in the population, then it is extremely difficult to encourage monolingual speaker of the mainstream language to study endangered language. Of course, it is also not a good measure for the protection of endangered language. How do we choose appropriate parameters such that the fractions of language $Y$ and bilingual $B$ are kept at reasonable levels with the minimal cost of control measure? It is an interesting problem; at the same time, it is extremely difficult.

\section{Concluding Remarks}

The dynamic behavior of two-language competitive model (1) with parameters (2) and $\alpha=1$ is analyzed systemically in this paper. By the linearization and Bendixson-Dulac theorem on dynamical system, some sufficient conditions on the globally asymptotical stability of the trivial equilibria, the existence, the local stability, and the global stability of positive equilibrium of model (1) are presented. The theoretical results show that languages $X$ and $Y$ are coexistent by adjusting the values of model parameters $k$ and $s$.

And when considering the protection of endangered language, model (1) with (2) is extended to model (10) assessing the impact of continuous control measure. The theoretical results and numerical simulations indicate that the existence and stability of model (10) are sensitive to control parameter. Furthermore, using the optimal control theory, we derived and analyzed the conditions for optimality of the endangered language. Our results say that the optimal control has a very desirable effect for maintaining the fraction 


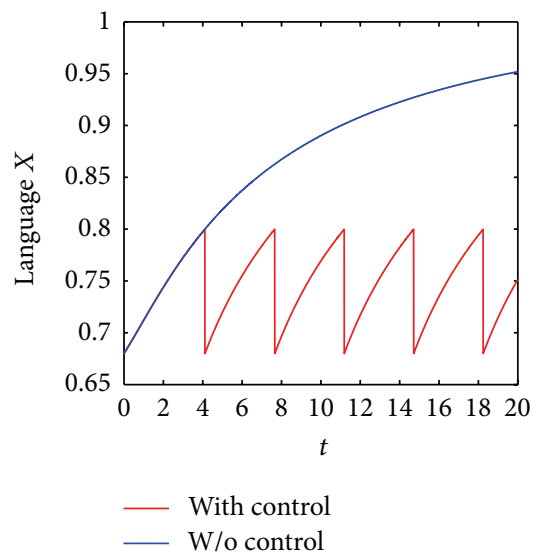

(a)

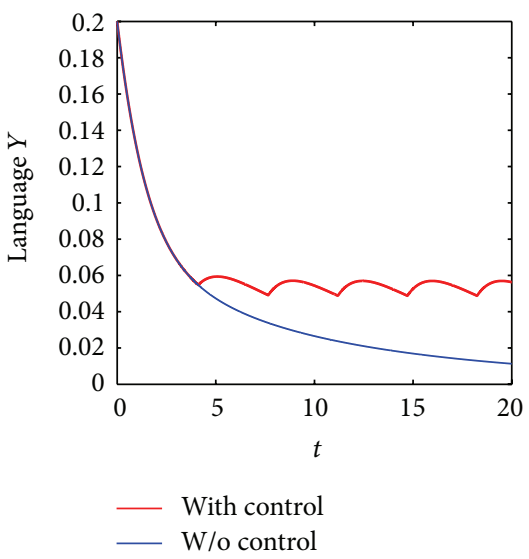

(b)

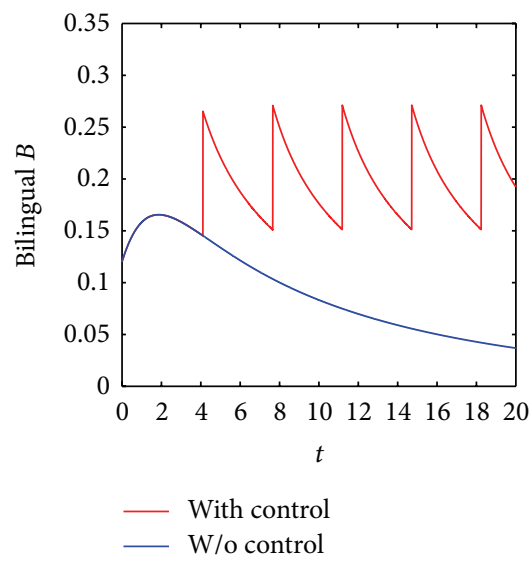

(c)

FIGURE 4: The contrast of the dynamical behaviors of model (29) with state-dependent impulsive control measure and without control, where $c=k=0.5, s=0.7, E_{X}=0.8$, and $\theta=0.15$.

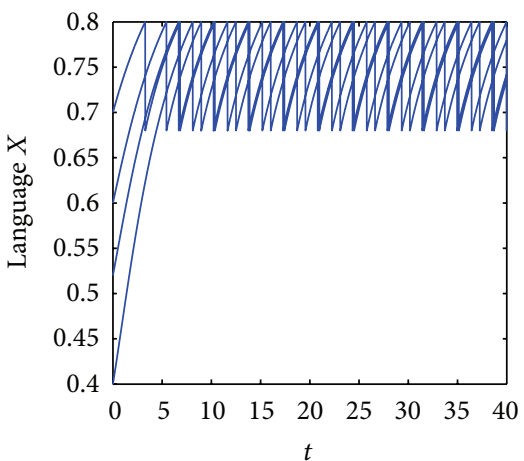

(a)

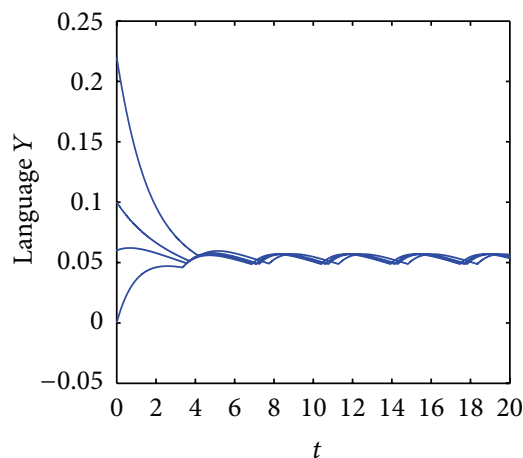

(b)

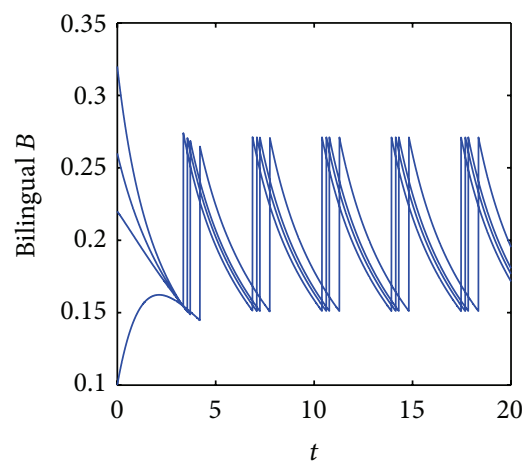

(c)

FIGURE 5: The orbitally asymptotical stability of positive order-1 periodic solution for model (29).

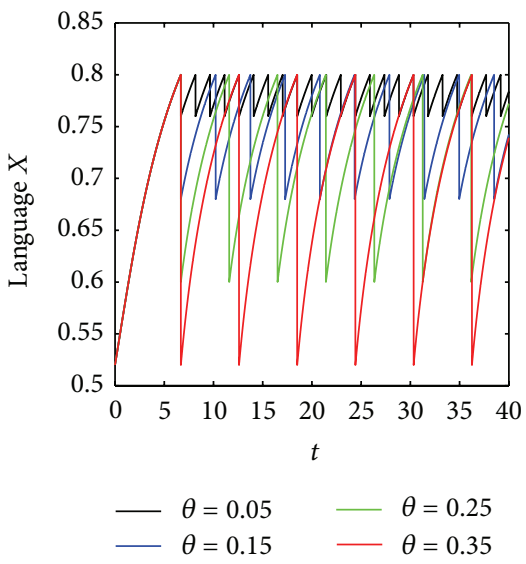

(a)

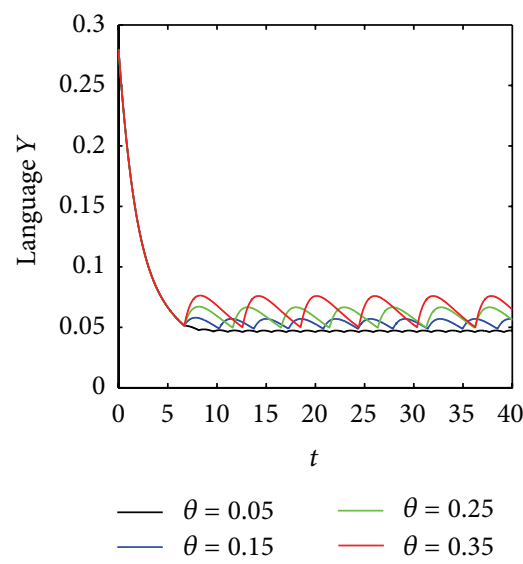

(b)

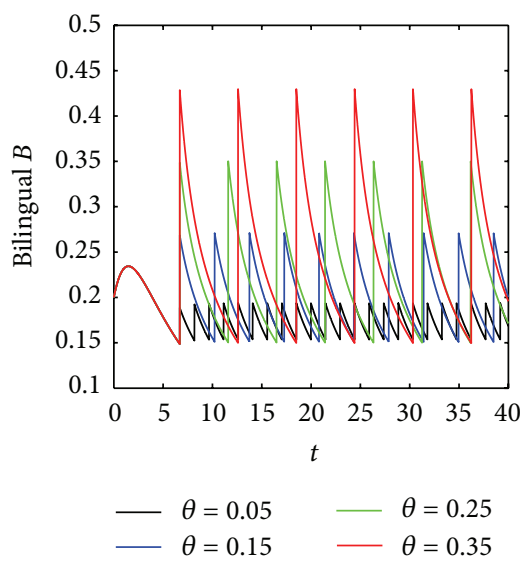

(c)

FIGURE 6: The contrast of the dynamical behaviors of model (29) with different state-dependent impulsive control parameters, where $E_{X}=0.8$ and $\theta=0.05,0.15,0.25$, and 0.35 , respectively. 


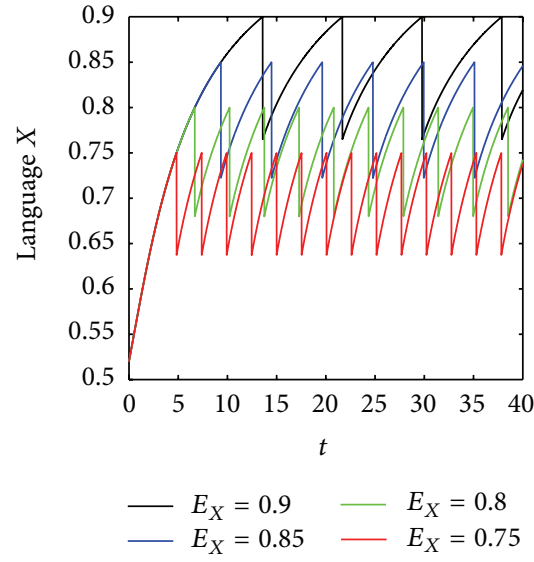

(a)

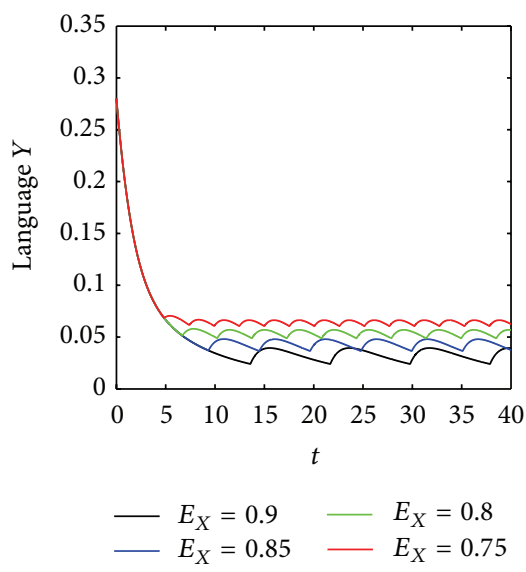

(b)

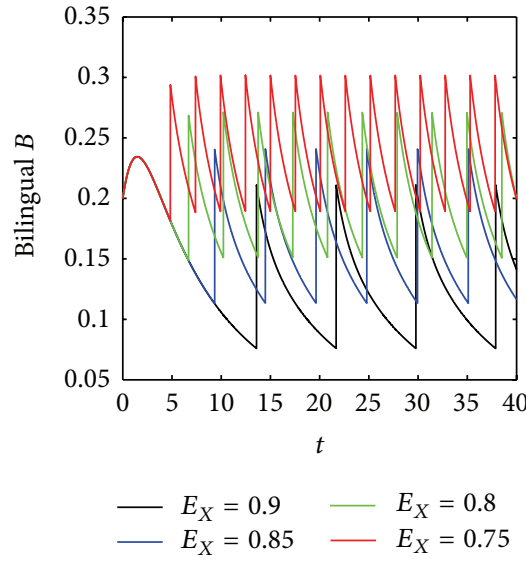

(c)

FIGURE 7: The contrast of the dynamical behaviors of model (29) with different state-dependent impulsive control parameters, where $\theta=0.15$ and $E_{X}=0.9,0.85,0.8$, and 0.75 , respectively.

of bilingual $B$, and some comparisons between with and without control are made in the figures.

Finally, the dynamic behavior of model (1) with statedependent pulse control measure, that is, model (29), is studied in Section 4. The state-dependent pulse control measure causes the complexity for the dynamic behavior of model (29) such as frequent switching between states, irregular motion, and some uncertainties. This is the distinguished feature compared with continuous control measure. By the Poincaré map, analogue of Poincaré criterion, and qualitative analysis method, some sufficient conditions on the existence and orbitally asymptotical stability of positive order-1 periodic solution are presented. This amounts to the fact that we can control the fractions of languages $X, Y$ and bilingual $B$ at reasonable levels by adjusting control parameters. Theoretical basis for finding a new measure to protect the endangered language is provided.

\section{Acknowledgments}

The authors wish to thank the reviewers and the handling editor, Prof. Bashir Ahmad, for their comments and suggestions, which led to a great improvement to the presentation of this work. This paper has been partially supported by the Scientific Research Programmes of Colleges in Xinjiang (Grant no. XJEDU2011S08), the Natural Science Foundation of Xinjiang (Grant no. 2011211B08), the National Natural Science Foundation of China (Grants nos. 11001235, 11271312, and 11261056), the China Postdoctoral Science Foundation (Grants nos. 20110491750 and 2012T50836), and the Ministerio de Economia y Competitividad (Spain), Project no. MTM2010-15314 and has been co-financed by the European Community Fund FEDER.

\section{References}

[1] W. J. Sutherland, "Parallel extinction risk and global distribution of languages and species," Nature, vol. 423, no. 6937, pp. 276$279,2003$.
[2] D. M. Abrams and S. H. Strogatz, "Modelling the dynamics of language death," Nature, vol. 424, no. 6951, p. 900, 2003.

[3] X. Castelló, V. M. Eguíluz, and M. San Miguel, "Ordering dynamics with two non-excluding options: Bilingualism in language competition," New Journal of Physics, vol. 8, article 308, 2006.

[4] I. Dyen, J. B. Kruskal, and P. Black, "An indoeuropean classication: a lexicostatistical experiment," American Philosophical Society, vol. 82, p. 5, 1992.

[5] T. Gong, L. Shuai, M. Tamariz, and G. Jäger, "Studying language change using Price equation and Pólya-urn dynamics," PLoS ONE, vol. 7, no. 3, Article ID e33171, 2012.

[6] J. A. Hawkins, "Innateness and function in language universals," in The Evolution of Human Languages, J. A. Hawkins and G. M. Murray, Eds., Addison-Wesley, Reading, Mass, USA, 1992.

[7] D. Helbing, A. Szolnoki, M. Perc, and G. Szabó, "Evolutionary establishment of moral and double moral standards through spatial interactions," PLoS Computational Biology, vol. 6, no. 4, Article ID e1000758, 2010.

[8] J. Mira and A. Plaredes, "Interlinguistic similarity and language death dynamics," Europhysics Letters, vol. 69, no. 6, pp. 10311034, 2005.

[9] M. A. Nowak, N. L. Komarova, and P. Niyogi, "Computational and evolutionary aspects of language," Nature, vol. 417, no. 6889, pp. 611-617, 2002.

[10] F. Petroni and M. Serva, "Language distance and tree reconstruction," Journal of Statistical Mechanics, vol. 2008, no. 8, Article ID P08012, 2008.

[11] C. Schulze, D. Stauffer, and S. Wichmann, "Birth, survival and death of languages by Monte Carlo simulation," Communications in Computational Physics, vol. 3, no. 2, pp. 271-294, 2008.

[12] M. Patriarca and E. Heinsalu, "Influence of geography on language competition," Physica A, vol. 388, no. 2-3, pp. 174-186, 2009.

[13] M. Patriarca and T. Leppänen, "Modeling language competition," Physica A, vol. 338, no. 1-2, pp. 296-299, 2004.

[14] D. Stauffer, X. Castelló, V. M. Eguíluz, and M. San Miguel, "Microscopic Abrams-Strogatz model of language competition," Physica A, vol. 374, no. 2, pp. 835-842, 2007. 
[15] J. Mira, L. F. Seoane, and J. J. Nieto, “The importance of interlinguistic similarity and stable bilingualism when two languages compete," New Journal of Physics, vol. 13, Article ID 033007, 2011.

[16] V. Otero-Espinar, L. F. Seoane, J. J. Nieto, and J. Mira, "An analytic solution of a model of language competition with bilingualism and interlinguistic similarity," Physica D, vol. 264, pp. 17-26, 2013.

[17] K. Blayneh, Y. Cao, and H.-D. Kwon, "Optimal control of vector-borne diseases: treatment and prevention," Discrete and Continuous Dynamical Systems B, vol. 11, no. 3, pp. 587-611, 2009.

[18] H. R. Joshi, "Optimal control of an HIV immunology model," Optimal Control Applications and Methods, vol. 23, no. 4, pp. 199-213, 2002.

[19] T. K. Kar and A. Batabyal, "Stability analysis and optimal control of an SIR epidemic model with vaccination," BioSystems, vol. 104, no. 2-3, pp. 127-135, 2011.

[20] D. Kirschner, S. Lenhart, and S. Serbin, "Optimal control of the chemotherapy of HIV," Journal of Mathematical Biology, vol. 35, no. 7, pp. 775-792, 1997.

[21] A. A. Lashari and G. Zaman, "Optimal control of a vector borne disease with horizontal transmission," Nonlinear Analysis: Real World Applications, vol. 13, no. 1, pp. 203-212, 2012.

[22] M. Ozari, A. A. Lashari, I. H. Jung, and K. O. Okosun, "Stability analysis and optimalcontrol of a Vector-Borne disease with nonlinear incidence," Discrete Dynamics in Nature and Society, vol. 2012, Article ID 595487, 21 pages, 2012.

[23] G. Zaman, Y. Han Kang, and I. H. Jung, "Stability analysis and optimal vaccination of an SIR epidemic model," BioSystems, vol. 93, no. 3, pp. 240-249, 2008.

[24] E. Jung, S. Lenhart, and Z. Feng, "Optimal control of treatments in a two-strain tuberculosis model," Discrete and Continuous Dynamical Systems B, vol. 2, no. 4, pp. 473-482, 2002.

[25] L. F. Nie, Z. D. Teng, and B. Z. Guo, "A state dependent pulse control strategy for a SIRS epidemic system," Bulletin of Mathematical Biology, vol. 75, no. 10, pp. 1697-1715, 2013.

[26] L. Nie, Z. Teng, and A. Torres, "Dynamic analysis of an SIR epidemic model with state dependent pulse vaccination," Nonlinear Analysis: Real World Applications, vol. 13, no. 4, pp. 16211629, 2012.

[27] S. Lenhart and J. T. Workman, Optimal Control Applied to Biological Models, Chapman \& Hall/CRC, London, UK, 2007.

[28] L. S. Pontryagin, V. G. Boltyanskii, R. V. Gamkrelidze, and E. F. Mishchenko, The Mathematical Theory of Optimal Process, vol. 14, Gordon \& Breach Science, New York, NY, USA, 1986.

[29] V. Lakshmikantham, D. D. Bainov, and P. S. Simeonov, Theory of Impulsive Differential Equations, World Scientific, Singapore, 1989.

[30] J. Hale and H. Kocak, Dynamics and Bifurcations, Springer, New York, NY, USA, 1991.

[31] P. S. Simeonov and D. D. Bainov, "Orbital stability of periodic solutions of autonomous systems with impulse effect," International Journal of Systems Science, vol. 19, no. 7, pp. 2561-2585, 1988. 


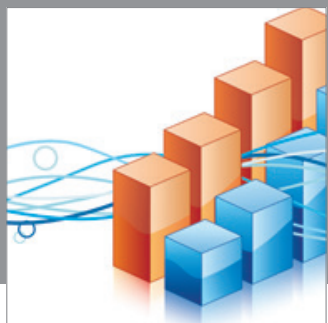

Advances in

Operations Research

mansans

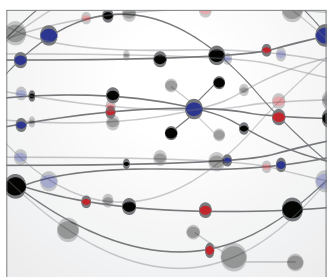

The Scientific World Journal
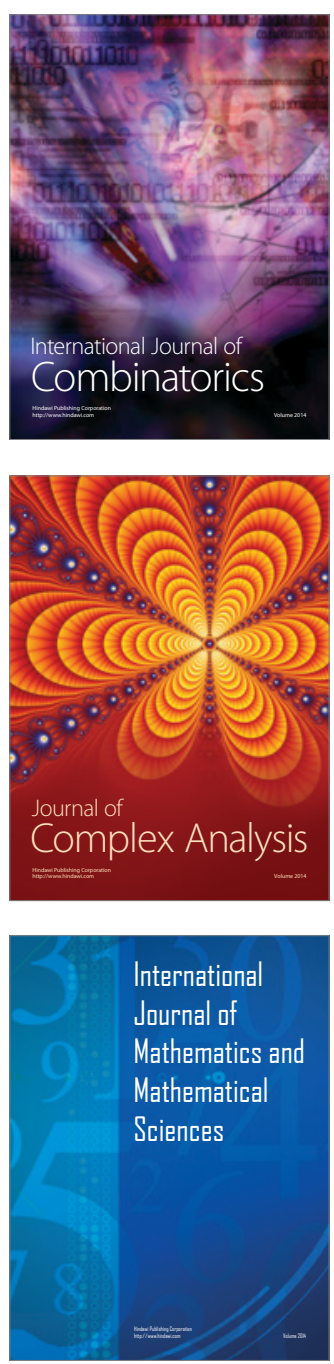
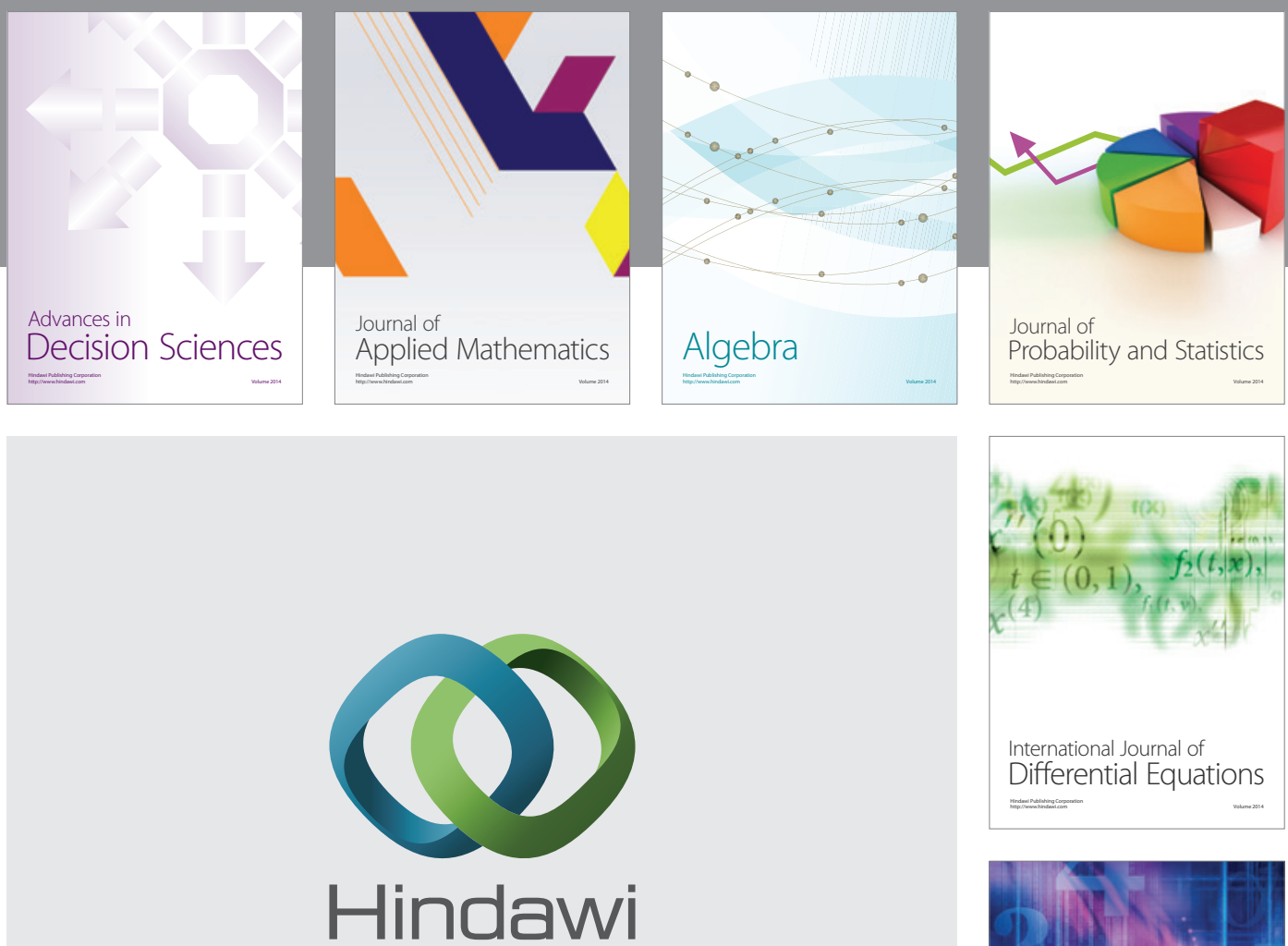

Submit your manuscripts at http://www.hindawi.com
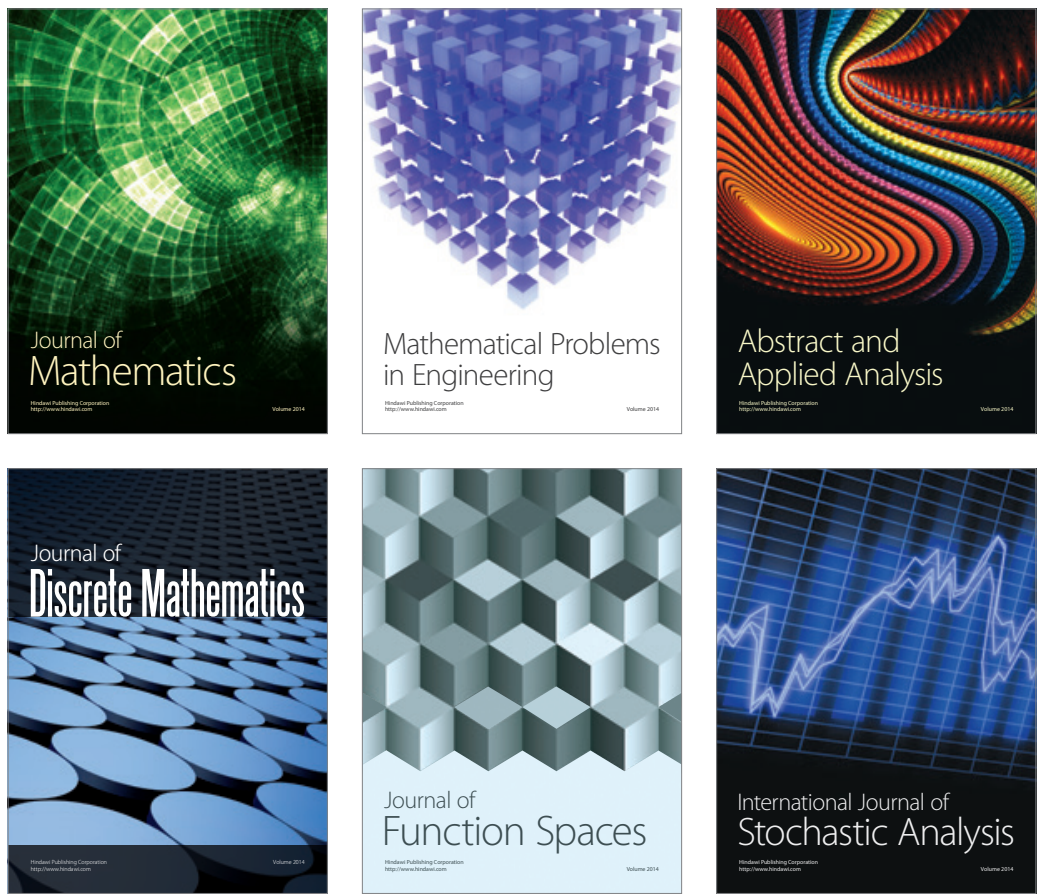

Journal of

Function Spaces

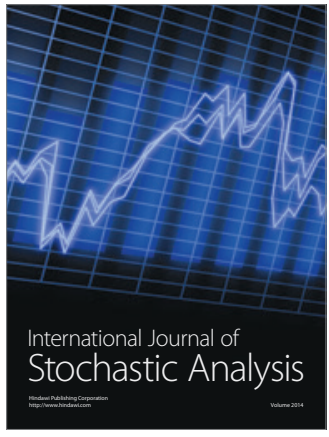

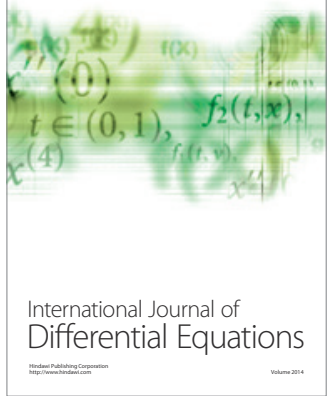
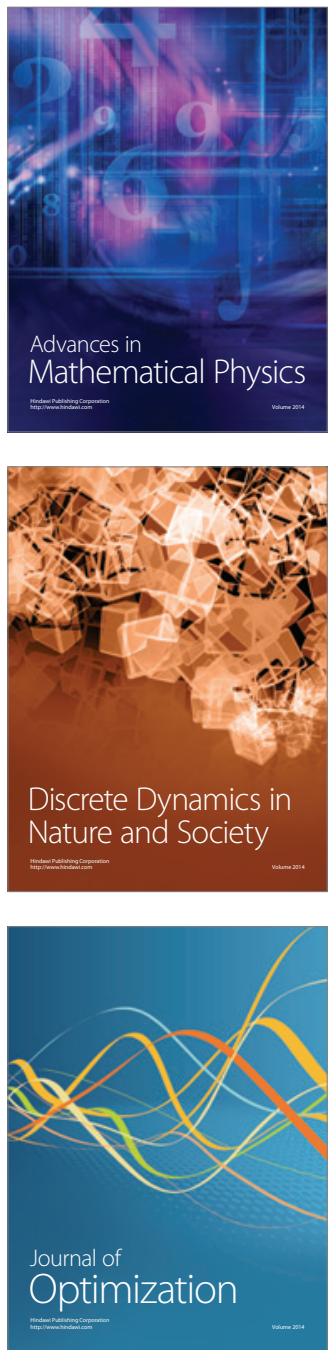